\title{
Molecular characterization of three intestinal protozoans in hospitalized children with different disease backgrounds in Zhengzhou, central China
}

\author{
Fuchang $\mathrm{Yu}^{1,2}$, Dongfang $\mathrm{Li}^{1,2}$, Yankai Chang ${ }^{1,2}$, Yayun $\mathrm{Wu}^{1,2}$, Zhenxin Guo ${ }^{3}$, Liting Jia ${ }^{4}$, Jinling $\mathrm{Xu}^{5}$, \\ Junqiang $\mathrm{Li}^{1,2,6}$, Meng $\mathrm{Qi}^{1,2,7}$, Rongjun Wang ${ }^{1,2}$ and Longxian Zhang ${ }^{1,2^{*}}$ (D)
}

\begin{abstract}
Background: Cryptosporidium spp. and Giardia duodenalis are major intestinal pathogens that can cause diarrheal diseases in humans, especially children. Enterocytozoon bieneusi is another parasite which can cause gastrointestinal tract disorders, with diarrhea being the main clinical symptom. However, few genetic studies of these parasites in pediatric inpatients in China have been published.

Methods: To assess the genetic characteristics and epidemiological status of these parasites, a total of 2284 fecal samples were collected from children in the pediatric departments of three hospitals in Zhengzhou, central China, and screened for these protozoans with PCR, based on the small subunit ribosomal RNA (SSU rRNA) genes of Cryptosporidium spp. and G. duodenalis and the internal transcribed spacer (ITS) of E. bieneusi.

Results: Six (0.26\%), 14 (0.61\%), and 27 (1.18\%) of the samples were positive for Cryptosporidium spp., G. duodenalis and E. bieneusi, respectively. Of the 12 successfully sequenced $G$. duodenalis isolates, four were identified as assemblage A and eight as assemblage B. In subtype and multilocus genotype (MLG) analyses, C. parvum IIdA19G1 $(n=4)$ and two novel G. duodenalis MLGs belonging to subassemblage All $(n=3)$ and BIV $(n=5)$ were successfully identified. The E. bieneusi isolates included genotypes $\mathrm{D}(n=17), \mathrm{J}(n=2)$, PigEBITS7 $(n=1), \mathrm{BEB} 6(n=1)$, and CM8 $(n=1)$. This is the first report of C. parvum subtype IIdA19G1 in HIV-negative children and E. bieneusi genotype CM8 in humans.

Conclusions: The dominance of zoonotic C. parvum subtype IIdA19G1 indicates that this parasite is turning into zoonotic origin from human-to-human transmission. The phylogenetic analysis also revealed the zoonotic origins and anthroponotic transmission potential of $\mathrm{G}$. duodenalis and E. bieneusi, suggesting more efforts must be made to minimize the threat these pathogens pose to public health.
\end{abstract}

Keywords: Cryptosporidium, Giardia duodenalis, Enterocytozoon bieneusi, Epidemiology, Children, China

\section{Background}

Cryptosporidium spp., Giardia duodenalis and Enterocytozoon bieneusi are the major etiological agents of cryptosporidiosis, giardiasis and microsporidiosis, respectively [1-3]. When infected with these parasites, although most

\footnotetext{
*Correspondence: zhanglx8999@henau.edu.cn; zhanglx8999@gmail.com ${ }^{1}$ College of Animal Science and Veterinary Medicine, Henan Agricultural University, Zhengzhou, Henan, People's Republic of China Full list of author information is available at the end of the article
}

of the immunocompetent individuals are always asymptomatic, there are also patients suffering self-limiting diarrhea or severe wasting disease, especially immunocompromised individuals, such as HIV-positive patients $[4,5]$. Susceptible hosts acquire infections through both direct and indirect transmission routes, such as direct contact or ingestion of contaminated food or water [5].

Molecular diagnostic tools based on the small subunit ribosomal RNA (SSU rRNA) gene of Cryptosporidium 
spp. and G. duodenalis and the internal transcribed spacer (ITS) of E. bieneusi have long been used for the molecular analysis of these parasites [3]. At least 36 valid species of Cryptosporidium have been reported worldwide [6], among which C. hominis and C. parvum are the Cryptosporidium species most frequently detected in human isolates [3]. Giardia duodenalis is considered a species complex, consisting of eight genetically distinct genotypes, assemblages $\mathrm{A}-\mathrm{H}$, which have different host specificities. Assemblages A and B appear to be responsible for most human infections $[7,8]$. Currently, over 1600 E. bieneusi ITS genotypes have been deposited in GenBank, derived from more than 650 studies [9]. Of these, genotypes D, EbpC, PigEBITS7 and type IV, are the genotypes most prevalent in humans [9].

According to the molecular epidemiological data available from many provinces in China (Jilin, Heilongjiang, Tianjin, Anhui, Hebei, Henan, Jiangsu, Shanghai, Hubei, Sichuan, Chongqing, Guangxi and Yunnan) [5, 10-12], the prevalence of Cryptosporidium ranges from $0.1 \%$ to $13.5 \%[13,14]$. Epidemiological investigations conducted in recent years have suggested that the average infection rate of G. duodenalis is $0.85 \%(197 / 23,098)$, and the highest infection rate $(9.46 \%, 7 / 74)$ was reported in one study in Shanghai $[10,15]$. The prevalence of E. bieneusi in humans varies from $0.2 \%$ to $22.5 \%$, with the highest infection rate observed in children in Jilin Province $[11,16]$.

Most published studies of these parasites have involved HIV-positive patients and children with diarrhea, but the infectious status of these parasites in children with different disease backgrounds remains unclear. Henan is a province located in central China, with a population of about 100 million. As the capital of Henan Province, Zhengzhou, has a population of more than 10 million and the public health threat in the event of an outbreak of these parasitic diseases is significant. Because the patients hospitalized in pediatric wards are by definition children, outbreaks of enteroparasites are more likely to occur when these children infected with highly virulent isolates. Therefore, we thoroughly assessed the prevalence and risk levels of Cryptosporidium spp., G. duodenalis and E. bieneusi in a large population of children hospitalized in Zhengzhou, Henan Province, central China.

\section{Methods}

\section{Sample collection}

Hospitals are ideal sites for surveying individuals with different disease backgrounds, and fecal samples can be readily collected. In this study, the pediatric departments of three hospitals in Zhengzhou, Henan Province, were selected to investigate the infection rates and genetic characteristics of Cryptosporidium, G. duodenalis and E. bieneusi in the children admitted to these hospitals.
All the parents or guardians were given specific oral instructions for the proper sample collection and the avoidance of possible contamination, and a plastic box labeled with a unique number in which to collect a fresh stool sample in the morning. A total of 2284 samples were collected from children aged 1-14 between March 2016 and January 2017, and all the samples were stored at $4{ }^{\circ} \mathrm{C}$ in $2.5 \%(\mathrm{w} / \mathrm{v})$ potassium dichromate solution until DNA extraction.

\section{DNA extraction and PCR amplification}

Dichromate was washed off the samples by distilled water and centrifugation. Genomic DNA was extracted from $\sim 200 \mathrm{mg}$ of each stool sample using the E.Z.N.A ${ }^{\circledR}$ stool DNA kit (Omega Bio-tek, Norcross, GA), according to the manufacturer's instructions. Extracted DNA preparations were analyzed by nested PCR amplification.

All samples were examined for Cryptosporidium spp. using a nested PCR that targeting a 834-bp fragment of the SSU rRNA gene $[17,18]$ The Cryptosporidium-positive samples were further analyzed with nested PCR targeting a 400-500-bp fragment of the $60 \mathrm{kDa}$ glycoprotein gene (gp60) [19]. All the samples were tested for G. duodenalis infection with nested PCR based on a 292-bp fragment of the $S S U$ rRNA, using a previously described assay [20]. Segments of the $\beta$-giardin gene ( $b g, \sim 510 \mathrm{bp})$, triose phosphate isomerase gene (tpi, $\sim 530 \mathrm{bp}$ ) and glutamate dehydrogenase gene $(g d h, \sim 530 \mathrm{bp})$ were then amplified with the method described by Sulaiman et al. [21], Cacciò et al. [22] and Lalle et al. [23] to determine the multilocus genotypes (MLGs) of the G. duodenalis isolates detected in this study. For the assemblage-specific amplification of the Giardia tpi gene, primers previously described by Geurden et al. [24] were used. The presence of E. bieneusi in the 2284 fecal samples was detected with nested PCR that amplified a 389-bp fragment of the ITS [25].

Reagent-grade water was used as the negative control for all PCRs and DNA known to be positive at the specific locus was used as the positive control. The amplified fragments were separated electrophoretically on $1 \%$ agarose gel stained with DNAGreen (Tiandz, Beijing, China) and visualized under UV light.

\section{Sequence and data analyses}

The positive secondary PCR products were sequenced bidirectionally on an ABI PRISM ${ }^{\mathrm{TM}} 3730$ XL DNA Analyzer by SinoGenoMax Biotechnology Co., Ltd (Beijing, China) using the BigDye Terminator v3.1 Cycle Sequencing Kit (Applied Biosystems, Foster City, CA, USA). The generated sequences were assembled and edited with DNASTAR Lasergene EditSeq version 7.1.0 (http://www. dnastar.com/) and aligned with reference sequences 
downloaded from GenBank with Clustal X version 2.1 (http://www.clustal.org/).

To infer the phylogenetic relationships of the detected samples, neighbor-joining (NJ) trees were constructed with the program MEGA X (http://www.megasoftwa re.net), based on evolutionary distances calculated with the Kimura 2-parameter model. The reliability of these trees was assessed with a bootstrap analysis of 1000 replicates.

All statistical analyses were performed with the software IBM SPSS Statistics (www.ibm.com/products/spssstatistics). The prevalence of parasitic infections, with the 95\% confidence interval (CI), was calculated. In the univariate analyses, Fisher's exact test was used to compare the prevalence of the three parasitic diseases in groups constructed according to age, sex, and disease background, and the odds ratios (ORs; with 95\% CIs) were computed to examine the associations between the participants' characteristics and the parasitic infections A two-sided $P$-value of 0.05 or less was deemed significant.

\section{Results}

\section{Occurrence of Cryptosporidium spp., G. duodenalis and $E$.} bieneusi

Of the 2284 fecal samples examined in this study from the three children's hospitals in Zhengzhou, six $(0.26 \%$, 95\% CI: $0.03-0.49 \%), 14$ (0.61\%, 95\% CI: $0.27-0.95 \%)$, and 27 (1.18\%, 95\% CI: 0.72-1.65\%) were positive for Cryptosporidium spp., G. duodenalis and E. bieneusi, respectively $(P=0.0007)$. No coinfections of these pathogens were detected in any of the samples. By age, the highest total infection rate $(5.22 \%, 14 / 268,95 \%$ CI: $2.37-8.07 \%)$ for all three parasites combined was detected in children aged 6-14 years, followed by children aged 1-6 years, with an infection rate of $2.48 \%$ (23/927, 95\% CI: $1.43-3.54 \%$ ); the lowest rate was $0.92 \%$
(10/1089, 95\% CI: $0.31-1.53 \%)$ in children aged $<1$ year $(P<0.0001)$ (Table 1$)$. The data showed that $2.12 \%(95 \%$ CI: $1.33-2.90 \%$ ) of males and 1.96\% (95\% CI: 0.98-2.94\%) $(P=0.88)$ of females were positive for an intestinal parasite. According to the children's disease backgrounds, the highest infection rate $(8.33 \%, 13 / 156,95 \%$ CI: $3.68-$ $12.99 \%)$ was observed in the group with gastrointestinal symptoms, followed by the group with autoimmune rheumatic disease $(4.98 \%, 11 / 221,95 \%$ CI: $1.88-8.07 \%)$ or hematological neoplasms $(4.14 \%, 6 / 145,95 \% \mathrm{CI}$ : $0.55-7.72 \%)$, whereas only $0.96 \%$ (17/1762, 95\% CI: $0.48-$ $1.45 \%)$ of the remaining 1762 samples from children with other medical conditions were positive $(P<0.0001)$ (Table 1).

\section{Correlation analysis}

The correlations between the clinical data and the infection rates were evaluated by computing the ORs and their 95\% CIs, which are shown in Table 1. There was a significant positive correlation between the infection rate and age in the hospitalized children, as an OR of 2.75 (95\% CI: $1.30-5.80 ; P=0.007)$ was associated with the $1-6$ yearold group and 5.97 (95\% CI: 2.61-13.54, $P<0.0001$ ) was associated with the 6-14 year-old group. No significant association between infection and sex $(\mathrm{OR}=0.92,95 \%$ CI: 0.51-1.68) was observed. The results of the univariate analysis also showed that children with gastrointestinal symptoms $(\mathrm{OR}=9.33,95 \% \mathrm{CI}$ : 4.44-19.60), autoimmune rheumatic disease $(\mathrm{OR}=5.38,95 \% \mathrm{CI}$ : $2.49-11.63)$, or hematological neoplasms ( $\mathrm{OR}=4.43,95 \% \mathrm{CI}: 1.72-$ $11.42)$ were at greatest risk of parasitic infection.

\section{Cryptosporidium species and gp60 subtypes}

The sequences of the SSU rRNA gene were obtained for five of the six positive samples, which showed that four of the patients were infected with C. parvum and one with

Table 1 Occurrence of three intestinal protozoans in children by age, sex and disease background

\begin{tabular}{lllll}
\hline Variable & No. of participants & Prevalence $(95 \% \mathrm{Cl})(\%)$ & $P$-value & OR (95\% Cl) \\
\hline $\begin{array}{l}\text { Age (years) } \\
<1\end{array}$ & 1089 & & $<0.0001$ & 1.00 \\
$1-6$ & 927 & $0.92(0.31-1.53)$ & 0.007 & $2.75(1.30-5.80)$ \\
$6-14$ & 268 & $2.48(1.43-3.54)$ & $<0.0001$ & $5.97(2.61-13.54)$ \\
Sex & & $5.22(2.37-8.07)$ & & 1.00 \\
Male & 1416 & $2.12(1.33-2.90)$ & 0.88 & $0.92(0.51-1.68)$ \\
$\quad$ Female & 868 & $1.96(0.98-2.94)$ & & \\
Disease background & & & $<0.0001$ & 1.00 \\
$\quad$ Other disease & 1762 & $0.96(0.48-1.45)$ & $<0.0001$ & $9.33(4.44-19.60)$ \\
$\quad$ Gastrointestinal disease & 156 & $8.33(3.68-12.99)$ & $<0.0001$ & $5.38(2.49-11.63)$ \\
Autoimmune rheumatic disease & 221 & $4.98(1.88-8.07)$ & 0.006 & $4.43(1.72-11.42)$ \\
Hematology neoplastic disease & 145 & $4.14(0.55-7.72)$ & & \\
\hline
\end{tabular}


Table 2 Occurrence and subtype, assemblage, or genotype distributions of Cryptosporidium spp., G. duodenalis and E. bieneusi detected in this study

\begin{tabular}{lll}
\hline Parasites & Prevalence (\%) & Subtype/assemblage/genotype $^{\text {a }}$ \\
\hline Cryptosporidium spp. & $0.26(n=6)$ & IdA19G1 $(n=4)$ \\
G. duodenalis & $0.61(n=14)$ & Assemblage A $(n=4) ;$ assemblage B $(n=8)$ \\
E. bieneusi & $1.18(n=27)$ & $\mathrm{D}(n=17) ; \mathrm{J}(n=2) ; \mathrm{PigEBITS7}(n=2) ; \mathrm{BEB} 6$ \\
& & $(n=1) ; \mathrm{CM} 8(n=1)$ \\
\hline
\end{tabular}

${ }^{a}$ Not all of the positive samples were sequenced successfully

C. hominis. All four C. parvum samples were subtyped as IIdA19G1R1, with no heterogeneity, whereas the single C. hominis sample was not typed successfully at the gp60 locus (Table 2).

Giardia duodenalis assemblages and multilocus genotypes Of the 14 PCR-positive samples of $G$. duodenalis at the $S S U$ rRNA locus, 12 were sequenced successfully. However, four were identified as assemblage A and eight as assemblage B. When the MLG technique was applied to these 14 samples, 11, 10 and 12 samples were positive at the $b g$, tp $i$ and $g d h$ loci, respectively (Tables 2,3 ).

A sequence analysis of the 11 sequences positive at the $b g$ locus showed that five isolates were assemblage A and six were assemblage $B$. The sequences from all the assemblage A isolates were identical to GenBank reference sequence MG736240. Within the assemblage B isolates, three sequences were identical to MG736242, whereas two sequences had a single-nucleotide polymorphism (SNP) (G389A or G239A) relative to the MG736242

Table 3 Multilocus genotyping of Giardia duodenalis isolates with a sequencing analysis of SSU rRNA, bg, tpi and gdh genes

\begin{tabular}{|c|c|c|c|c|c|}
\hline \multirow[t]{2}{*}{ Isolate } & \multicolumn{4}{|c|}{ G. duodenalis assemblage } & \multirow[t]{2}{*}{ MLGs } \\
\hline & SSU rRNA & $b g$ & tpi & $g d h$ & \\
\hline FY86 & B & $\mathrm{B}$ & $B$ & B & BIV-Novel1 \\
\hline FY120 & SN & A & PN & B & \\
\hline ET91 & B & B & B & $B$ & BIV-Novel2 \\
\hline ET129 & $\mathrm{B}$ & $B$ & $\mathrm{~B}$ & B & BIV-Novel3 \\
\hline ET131 & B & B & $\mathrm{B}$ & $\mathrm{B}$ & BIV-Novel3 \\
\hline ET265 & A & A & $A$ & A & All-Novel1 \\
\hline ET346 & A & A & $A$ & A & All-Novel2 \\
\hline ED127 & B & B & $\mathrm{B}$ & B & BIV-Novel3 \\
\hline ED158 & B & PN & B & PN & \\
\hline ED221 & $B$ & B & PN & PN & \\
\hline ED303 & $A$ & A & SN & $A$ & \\
\hline ED421 & $A$ & $A$ & $A$ & A & All-Novel2 \\
\hline ED462 & SN & PN & PN & B & \\
\hline ED551 & B & SN & A & $A+B^{a}$ & \\
\hline
\end{tabular}

Abbreviations: PN, PCR-negative; SN, sequence-negative

a Results of forward sequencing showed assemblage $B$, whereas reverse sequencing showed assemblage $A$ sequence, and the remaining sequence had two SNPs (A95G and C188T).

According to the sequence analysis of the $g d h$ locus, four isolates were identified as assemblage A and seven as assemblage B. Isolate ED551 showed inconsistent bidirectional sequencing results, because the forward sequence identified it as assemblage $B$, whereas the reverse sequence identified it as assemblage A. All four assemblage A sequences and the reverse sequence of isolate ED551 were consistent with KT948091. Apart from the forward sequence of isolate ED551, the sequences obtained for the assemblage B isolates were identical to EU637585, except one sequence with one SNP (T318C) relative to $\mathrm{MH} 475908$.

At the tpi locus, assemblages A and B were identified in four and six PCR-positive samples, respectively. One and three assemblage A sequences were identical to reference sequences MH673809 and MH673818, respectively. Among the assemblage B sequences, two sequences were consistent with KT948104 or KX668308, and the remaining four sequences showed one SNP (G250A) relative to KF679742. Because of its inconsistent bidirectional sequencing results at the $g d h$ locus, isolate ED551 was amplified and sequenced again using assemblage $\mathrm{A}$ and assemblage B-specific primers of the tpi gene, respectively. Interestingly, a mixed infection of assemblage A and B was detected in ED551.

Because of its inconsistent bidirectional sequencing results, ED551 was excluded from the MLG analysis, as were those samples for which data for all three loci were not available. In total, five MLGs were generated (two belonging to assemblage A and three belonging to assemblage B) and were designated AII-Novel1, AII-Novel2, and BIV-Novel1 to BIV-Novel3. All the MLGs were found in a single isolate, except BIV-Novel3, which occurred in three isolates (Table 3). In a phylogenetic analysis, the two assemblage A MLGs clustered closely with MLG AII-1 (Fig. 1) and the three assemblage B MLGs clustered on the MLG BIV branch (Fig. 2).

\section{Enterocytozoon bieneusi genotypes}

Of the 27 samples positive for E. bieneusi according to PCR amplification of the ITS fragment, 23 were 


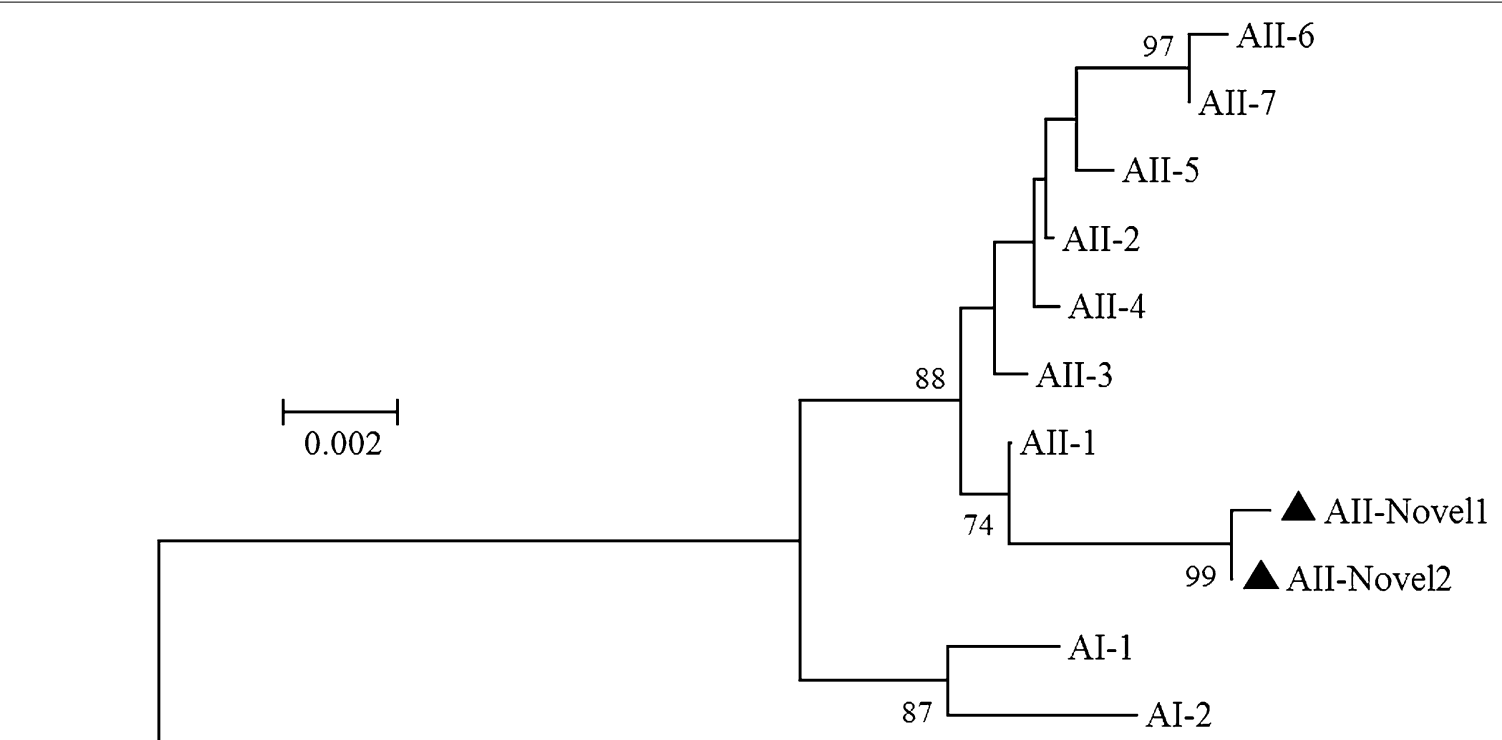

AIII-1

Fig. 1 Phylogenetic relationships among G. duodenalis assemblage A isolates inferred with a neighbor-joining analysis based on the concatenated bg, tpi and gdh nucleotide sequences. Multilocus genotypes from previous studies were used as the reference sequences and are represented by the corresponding isolate name [22]. Novel MLGs are indicated with triangles. Bootstrap values $>70 \%$ from 1000 replicates are shown at the nodes

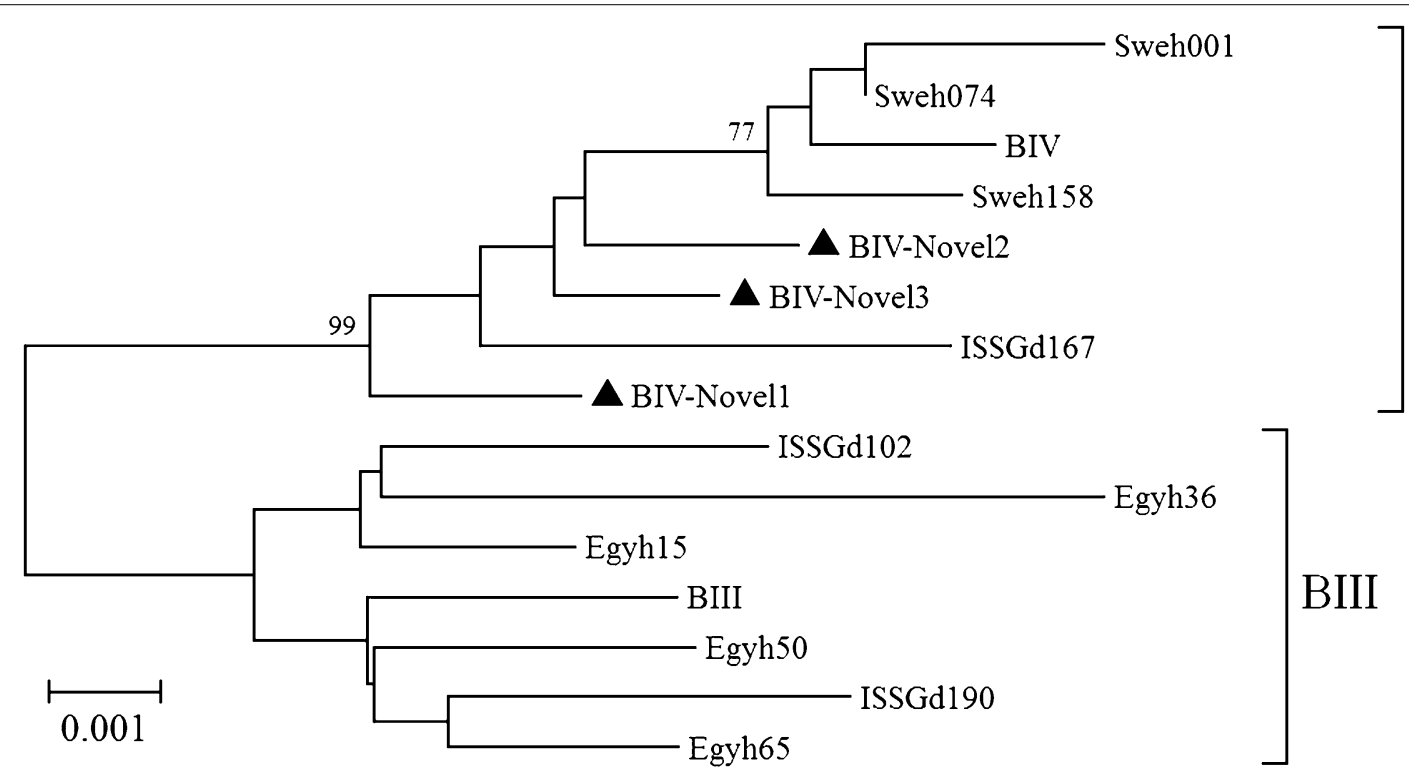

Fig. 2 Phylogenetic relationships among G. duodenalis assemblage B isolates inferred with a neighbor-joining analysis based on the concatenated bg, tpi and gdh nucleotide sequences. Multilocus genotypes from previous studies were used as the reference sequences and are represented by the corresponding isolate names $[8,22,47]$. Novel MLGs are indicated with triangles. Bootstrap values $>70 \%$ from 1000 replicates are shown at the nodes

sequenced successfully. Sequence analysis identified five genotypes: D, J, PigEBITS7, BEB6 and CM8. D was the dominant genotype and was detected in 17 isolates, whereas the other genotypes were restricted to one or two isolates (Table 2). In a phylogenetic analysis, three of the five genotypes identified in this study (D, CM8 and PigEBITS7) clustered in group 1 and genotypes $J$ and BEB6 clustered in group 2 (Fig. 3). 


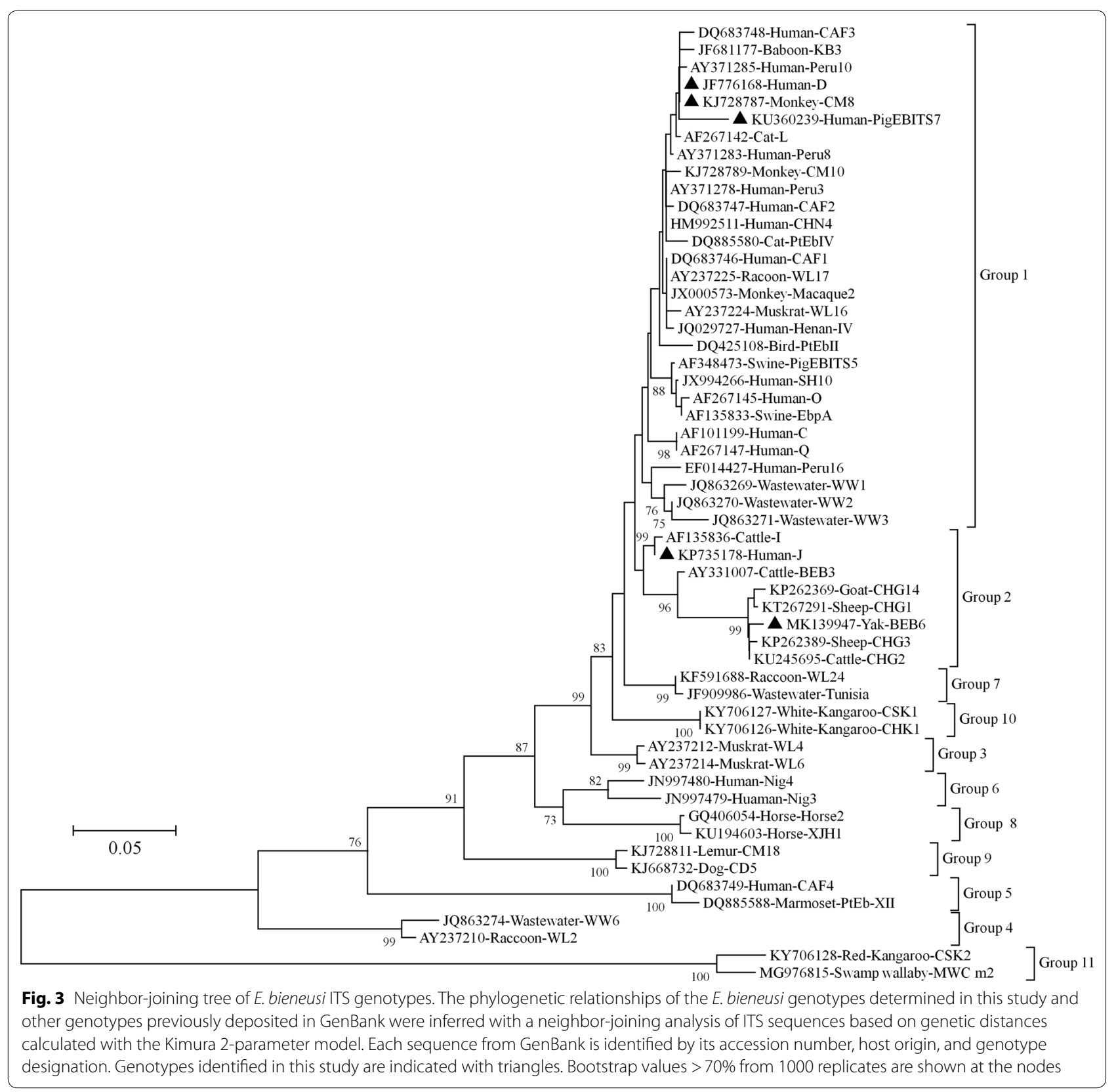

\section{Discussion}

Our data indicate that children aged 6-14 years were more likely to be infected with enteroparasites than those aged $<1$ year or 1-6 years, suggesting that children aged 6-14 years are at highest risk of infection by these parasites, which is inconsistent with previous studies [26, 27]. These discrepancies might reflect the socioeconomic levels of the study sites, the immune status of the targeted groups, and/or the sensitivity of the techniques used [28]. In this study, newborns under one month of age were also included as long as they met the criteria. In the traditional Chinese concept of infant care, these newborns have little contact with anything other than their mothers during the first month of their lives. In one of the three hospitals involved in this study, the majority of pediatric inpatients were neonates, among whom no parasitic infections were found, and this may be contributed to the low infection rates of these parasites. Interestingly, higher infection rates were found in children with gastrointestinal symptoms, autoimmune rheumatic disease, or hematological neoplasms than in children with other diseases, which might be explained by the nature of these parasites, which cause gastroenteric symptoms, especially in immunocompromised patients [1-3]. The innate 
immune response plays an important role in the pathogenesis of autoimmune rheumatic disease, and many inhibitors are used today to treat this disease, including interleukin 6 (IL-6) inhibitors, IL-1 inhibitors, Janus kinase inhibitors and anti-B cell drugs [29]. Therefore, the immune function of the patient is inhibited to some extent, which may explain the high infection levels in the children with autoimmune rheumatic disease.

The prevalence of Cryptosporidium spp. (0.26\%) was consistent with previous molecular epidemiological studies of Cryptosporidium in children in other cities and provinces of China (1.2\% in Taiwan, $0.1 \%$ in Kunming, $2.0 \%$ in Wuhan, and $1.6 \%$ in Shanghai) [13, 30-32]. In a previous study conducted in Henan, the infection rate of Cryptosporidium was $1.5 \%$, but it was $0.15 \%$ in $683 \mathrm{HIV}$ positive patients in the National Free Antiretroviral Therapy Programme (NFATP) in China [33]. Cryptosporidium was detected in $0.11 \%$ of the samples in a cross-sectional study of 1637 children aged 3-9 years in Jiangsu [28]. In Sichuan Province, the infection rate of Cryptosporidium was reported to be $2.4 \%$ in a school-based cross-sectional study involving 321 elementary school children [34].

In our sequence analysis of the positive PCR products, C. parvum predominated, and was detected in four of the five successfully sequenced Cryptosporidium-positive samples. Inconsistent results have been published in previous studies. In a genetic characteristic analysis conducted in Henan, C. hominis was identified in nine of 10 Cryptosporidium spp. isolates from human samples [35]. Similarly, C. hominis accounted for $90.2 \%$ (92/102) of Cryptosporidium spp. infections in patients in Shanghai [31]. When subtyped at the gp60 locus, all the four C. parvum isolates detected in the present study were identified as IIdA19G1, a subtype which has recently been reported in several studies of human samples worldwide. For example, two $C$. parvum isolates derived from HIV-positive patients in Henan, China, were identified as IIdA19G1 [33]. This subtype has also been reported in human samples in Madrid, Spain [36] and in Portugal [37]. IIdA19G1 is also one of the most commonly detected subtypes in calves in Henan, Hebei, Beijing, and Heilongjiang Provinces [38-42] in horses in Sichuan, and in donkeys in Henan and Shandong Provinces [43]. Cryptosporidium parvum has been reported more frequently than $C$. hominis in most recent studies in China, suggesting a zoonotic origin rather than its anthroponotic transmission. This increasingly dominant transmission route of this parasite might be attributable to the rapid development of intensive breeding in recent decades and has important implications for public-health strategies to control these parasites.
In the present study, the infection rate of $G$. duodenalis was $0.61 \%$. A large number of epidemiological investigations have been conducted in China since the start of this century, and have suggested that the average infection rate of $G$. duodenalis is $0.85 \%(197 / 23,098)$ [10]. Our data are consistent with a more recent study in Wuhan, where a low infection rate (1.4\%) of G. duodenalis was detected [32]. In another study, G. duodenalis was detected in 17 of 252 fecal samples collected from patients with diarrhea in Shanghai, with an infection rate of $6.75 \%$, which is higher than that in the present study [44]. A similar observation was also made in India, where a high rate of $G$. duodenalis infection (10.2\%, 413/4039) was seen throughout the study period across all climatic conditions [45]. The infection rate of G. duodenalis is considered to be influenced by many factors, including ecological, environmental, and demographic conditions, and especially the immune status of the host [46].

In the MLG analysis of the G. duodenalis isolates, five MLGs were detected when the sequences at the $b g, t p i$ and $g d h$ loci were combined. In a phylogenetic analysis, two MGLs belonged to subassemblage AII and the remaining five MLGs clustered with subassemblage BIV. Subassemblages AII and BIV are the MLGs found most frequently in human samples in China [1, 15, 32, 35], suggesting an important role for anthroponotic transmission in the epidemiology of giardiasis. BIV was the most prevalent MLG in the present study, identified in $62.5 \%(5 / 8)$ of the G. duodenalis isolates, whereas BIII was found in none. However, different results have been observed in one of our previous study [47] and in a study conducted by Naguib et al. [48], in which an equal proportion of subassemblage BIII and BIV MLGs were reported. These discrepant results confirm the geographic segregation of the distributions of subassemblages BIII and BIV.

Of the fecal samples tested with PCR, 27 (1.18\%) were positive for $E$. bieneusi infection. In China, the prevalence of $E$. bieneusi in humans varies from $0.2 \%$ to $22.5 \%$ $[16,32,33,49]$, and a similar infection rate $(1.3 \%, 5 / 381)$ was detected in a previous study, in which 381 stool samples from cancer patients in China were screened [50]. As well as the immune status of the host, E. bieneusi infection rates are associated with many factors, including but not limited to the economic status and living conditions of the subjects [50].

In an analysis of the ITS sequences of E. bieneusi, D was the most common genotype, detected in 17 samples. According to the few reports of this parasite in humans in China, genotype D has been detected in children with diarrhea, HIV-positive patients, and HIV-negative individuals in Shanghai, Henan, Wuhan and Guangxi [15, $32,33,51]$. Genotype D has also been detected in at least 
15 animal species, including companion animals, livestock, wildlife, rodents, and birds $[9,50]$. In one of our recent studies, this genotype was found in synanthropic rodents living on a dairy farm [52]. Genotype D has also been observed in river water and wastewater [9], and these data draw a concise profile of the zoonotic potential of E. bieneusi genotype $\mathrm{D}$, which is transmitted via the indirect fecal-oral route. Genotypes PigEBITS7, J, and BEB6 may also be transmitted between humans and animals, because many researchers have reported human infections of these genotypes in China, which were first detected in animal samples $[15,16,33,51]$. Interestingly, to the best of our knowledge, this is the first report of a human infection of genotype CM8, which was first found in non-human primates by Karim et al. [53]. It has since been shown to infect dairy cattle [54] and horses [55]. These data extend the host range of this genotype and demonstrate its zoonotic potential.

\section{Conclusions}

We have shown low burdens of Cryptosporidium spp., G. duodenalis and E. bieneusi in children hospitalized in the pediatric departments of three hospitals in Zhengzhou, central China. A univariate analysis showed that children aged 6-14 years are most likely to be infected by these parasites, as are children with gastrointestinal symptoms, autoimmune rheumatic disease, or hematological neoplasms. The dominance of C. parvum subtype IIdA19G1 suggests the zoonotic origin of this parasite in children involved in this study. The occurrence of G. duodenalis subassemblages AII and BIV suggests the anthroponotic transmission of $G$. duodenalis and confirms the geographic segregation in the distributions of the different G. duodenalis subassemblages. The five E. bieneusi genotypes identified here have all been isolated from humans and a vast range of animal species, indicating that the zoonotic transmission of E. bieneusi is possible. Therefore, more holistic and integrated approaches must be used to minimize the threat posed by these parasitic pathogens to public health.

\section{Abbreviations \\ SSU rRNA: small subunit ribosomal RNA; ITS: internal transcribed spacer; HIV: human immunodeficiency virus; gp60: the $60 \mathrm{kDa}$ glycoprotein gene; $\mathrm{Cl}$ : con- fidence interval; OR: odds ratio; SNP: single-nucleotide polymorphism; MLG: multilocus genotype; IL: interleukin; NFATP: the National Free Antiretroviral Therapy Programme.}

\section{Acknowledgements}

We thank Janine Miller from Liwen Bianji, Edanz Editing China (http://www. liwenbianji.cn/ac), for editing the English text of a draft of this manuscript.

\section{Authors' contributions}

FY, DL, YC, YW, ZG, L and JX collected samples. FY, JL, MQ and RW performed analysis and interpretation. $F Y, J L$, and $M Q$ wrote the first draft of the manuscript. RW and LZ supervised the project. LZ obtained grant funding and wrote the final version of the paper. All authors read and approved the final manuscript.

\section{Funding}

The study was partly supported by the National Key Research and Development Programme of China (2017YFD0500405, 2017YFD0501305), the National Natural Science Foundation of China $(30600603,31672548)$, and the Natural Science Foundation of Henan Province (162300410129).

\section{Availability of data and materials}

Data supporting the conclusions of this article are included within the article. The newly generated sequences were deposited in the GenBank database under the accession numbers MK962813-MK962826, MK990042, MK990043 and MK990733-MK990737.

\section{Ethics approval and consent to participate}

All research involving human participants was approved by the Ethics Review Committee of Henan Agricultural University (Approval No. IRCHENAU-20160224-05). Written informed consent for participation in the study was obtained from the parent or guardian of each child after they were informed of the purposes and procedures of the study by medical workers. The authors declare that all the procedures used in this work complied with Declaration of Helsinki 1975, as revised in 2013. Participants infected with parasites received the appropriate treatment or referrals, according to the local treatment policies and national guidelines.

\section{Consent for publication}

Not applicable.

\section{Competing interests}

The authors declare that they have no competing interests.

\section{Author details}

${ }^{1}$ College of Animal Science and Veterinary Medicine, Henan Agricultural University, Zhengzhou, Henan, People's Republic of China. ${ }^{2}$ National Joint Research Center for Animal Immunology, Zhengzhou, Henan, People's Republic of China. ${ }^{3}$ Zhengzhou Children's Hospital, Zhengzhou, Henan, People's Republic of China. ${ }^{4}$ The Third Affiliated Hospital of Zhengzhou University, Zhengzhou, Henan, People's Republic of China. ${ }^{5}$ Henan Province People's Hospital, Zhengzhou, Henan, People's Republic of China. ${ }^{6}$ Scientific Research Experiment Center \& Laboratory Animal Center, Henan University of Chinese Medicine, Zhengzhou 450046, People's Republic of China. ${ }^{7}$ College of Animal Science, Tarim University, Alar, Xinjiang, People's Republic of China.

Received: 31 July 2019 Accepted: 10 November 2019

Published online: 15 November 2019

\section{References}

1. Feng Y, Xiao L. Zoonotic potential and molecular epidemiology of Giardia species and giardiasis. Clin Microbiol Rev. 2011;24:110-40.

2. Beatriz L, Isabel L, Cristina A, Soledad F, Julio T, Del Aguila C. Intestinal microsporidiosis due to Enterocytozoon bieneusi in elderly human immunodeficiency virus-negative patients from Vigo, Spain. Clin Infect Dis. 2002;34:918-21.

3. Xiao L. Molecular epidemiology of cryptosporidiosis: an update. Exp Parasitol. 2010;124:80-9.

4. Li J, Qi M, Chang Y, Wang R, Li T, Dong H, et al. Molecular characterization of Cryptosporidium spp, Giardia duodenalis, and Enterocytozoon bieneusi in captive wild life at Zhengzhou Zoo, China. J Eukaryot Microbiol. 2015;62:833-9.

5. Wang R, Li J, Chen Y, Zhang L, Xiao L. Widespread occurrence of Cryptosporidium infections in patients with HIV/AIDS: epidemiology, clinical feature, diagnosis, and therapy. Acta Trop. 2018;187:257-63.

6. Čondlová Š, Horčičková M, Sak B, Květoňová D, Hlásková L, Konečný R, et al. Cryptosporidium apodemi sp. n. and Cryptosporidium ditrichi sp. 
n. (Apicomplexa: Cryptosporidiidae) in Apodemus spp. Eur J Protistol. 2018;63:1-12.

7. Cacciò SM, Lalle M, Svärd SG. Host specificity in the Giardia duodenalis species complex. Infect Genet Evol. 2018;66:335-45.

8. Lebbad M, Petersson I, Karlsson L, Botero-Kleiven S, Andersson JO, Svenungsson B, et al. Multilocus genotyping of human Giardia isolates suggests limited zoonotic transmission and association between assemblage B and flatulence in children. PLoS Negl Trop Dis. 2011;5:e1262.

9. Li W, Feng Y, Santin M. Host specificity of Enterocytozoon bieneusi and public health implications. Trends Parasitol. 2019;35:436-51.

10. Li J, Wang H, Wang R, Zhang L. Giardia duodenalis infections in humans and other animals in China. Front Microbiol. 2017;8:2004.

11. Qiu L, Xia W, Li W, Ping J, Ding S, Liu H. The prevalence of microsporidia in China: a systematic review and meta-analysis. Sci Rep. 2019;9:3174.

12. Wang S, Wang R, Fan X, Liu T, Zhang L, Zhao G. Prevalence and genotypes of Enterocytozoon bieneusi in China. Acta Trop. 2018;183:142-52.

13. Hung CC, Tsaihong JC, Lee YT, Deng HY, Hsiao WH, Chang SY, et al. Prevalence of intestinal infection due to Cryptosporidium species among Taiwanese patients with human immunodeficiency virus infection. J Formos Med Assoc. 2007;106:31-5.

14. Mahmoudi MR, Ongerth JE, Karanis P. Cryptosporidium and cryptosporidiosis: the Asian perspective. Int J Hyg Environ Health. 2017:220:1098-109.

15. Wang L, Xiao L, Duan L, Ye J, Guo Y, Guo M, et al. Concurrent infections of Giardia duodenalis, Enterocytozoon bieneusi, and Clostridium difficile in children during a cryptosporidiosis outbreak in a pediatric hospital in China. PLoS Negl Trop Dis. 2013;7:2437.

16. Zhang X, Wang Z, Su Y, Liang X, Sun X, Peng S, et al. Identification and genotyping of Enterocytozoon bieneusi in China. J Clin Microbiol. 2011;49:2006-8.

17. Jiang J, Alderisio KA, Xiao L. Distribution of cryptosporidium genotypes in storm event water samples from three watersheds in New York. Appl Environ Microbiol. 2005;71:4446.

18. Xiao L, Morgan UM, Limor J, Escalante A, Arrowood M, Shulaw W, et al. Genetic diversity within Cryptosporidium parvum and related Cryptosporidium species. Appl Environ Microbiol. 1999;65:3386-91.

19. Essid R, Chelbi H, Siala E, Bensghair I, Menotti J, Bouratbine A. Polymorphism study of Cryptosporidium hominis gp60 subtypes circulating in Tunisia. Microb Pathog. 2017;110:298-303.

20. Appelbee AJ, Frederick LM, Heitman TL, Olson ME. Prevalence and genotyping of Giardia duodenalis from beef calves in Alberta, Canada. et Parasitol. 2003;112:289-94.

21. Sulaiman IM, Fayer R, Bern C, Gilman RH, Trout JM, Schantz PM, et al. Triosephosphate isomerase gene characterization and potential zoonotic transmission of Giardia duodenalis. Emerg Infect Dis. 2003;9:1444-52.

22. Cacciò SM, Beck R, Lalle M, Marinculic A, Pozio E. Multilocus genotyping of Giardia duodenalis reveals striking differences between assemblages A and B. Int J Parasitol. 2008;38:1523-31.

23. Lalle M, Pozio E, Capelli G, Bruschi F, Crotti D, Cacciò SM. Genetic heterogeneity at the beta-giardin locus among human and animal isolates of Giardia duodenalis and identification of potentially zoonotic subgenotypes. Int J Parasitol. 2005;35:207-13.

24. Geurden T, Levecke B, Cacciò SM, Visser A, De Groote G, Casaert S, et al. Multilocus genotyping of Cryptosporidium and Giardia in non-outbreak related cases of diarrhoea in human patients in Belgium. Parasitology. 2009;136:1161-8.

25. Sulaiman IM, Fayer R, Yang C, Santin M, Matos O, Xiao L. Molecular characterization of Enterocytozoon bieneusi in cattle indicates that only some isolates have zoonotic potential. Parasitol Res. 2004;92:328-34.

26. Al-Delaimy AK, Al-Mekhlafi HM, Nasr NA, Sady H, Atroosh WM, Nashiry M, et al. Epidemiology of intestinal polyparasitism among Orang Asli school children in rural Malaysia. PLoS Negl Trop Dis. 2014;8:e3074.

27. Al-Mohammed HI, Amin TT, Aboulmagd E, Hablus HR, Zaza BO. Prevalence of intestinal parasitic infections and its relationship with sociodemographics and hygienic habits among male primary school children in Al-Ahsa, Saudi Arabia. Asian Pac J Trop Med. 2010;3:906-12.

28. Zheng H, He J, Wang L, Zhang R, Ding Z, Hu W. Risk factors and spatial clusters of Cryptosporidium infection among school-age children in a rural region of eastern China. Int J Env Res Public Heal. 2018;15:924.
29. Conigliaro P, Triggianese P, De Martino E, Chimenti MS, Sunzini F, Viola A, et al. Challenges in the treatment of rheumatoid arthritis. Autoimmun Rev. 2019;18:706-13.

30. Zhang SX, Zhou YM, Xu W, Tian LG, Chen JX, Chen SH, et al. Impact of co-infections with enteric pathogens on children suffering from acute diarrhea in southwest China. Infect Dis Poverty. 2016;5:64.

31. Feng Y, Wang L, Duan L, Gomez-Puerta LA, Zhang L, Zhao X, et al. Extended outbreak of cryptosporidiosis in a pediatric hospital, China. Emerg Infect Dis. 2012;18:312-4.

32. Wang T, Fan Y, Koehler AV, Ma G, Li T, Hu M, et al. First survey of Cryptosporidium, Giardia and Enterocytozoon in diarrhoeic children from Wuhan. China. Infect Genet Evol. 2017;51:127-31.

33. Wang L, Zhang H, Zhao X, Zhang L, Zhang G, Guo M, et al. Zoonotic Cryptosporidium species and Enterocytozoon bieneusi genotypes in HIV-positive patients on antiretroviral therapy. J Clin Microbiol. 2013;51:557-63.

34. Yang D, Yang Y, Wang Y, Yang Y, Dong S, Chen Y, et al. Prevalence and risk factors of Ascaris lumbricoides, Trichuris trichiura and Cryptosporidium infections in elementary school children in southwestern China: a schoolbased cross-sectional study. Int J Env Res Pub Health. 2018;15:1809.

35. Wang R, Zhang X, Zhu H, Zhang L, Feng Y, Jian F, et al. Genetic characterizations of Cryptosporidium spp. and Giardia duodenalis in humans in Henan. China. Exp Parasitol. 2011;127:42-5.

36. de Lucio A, Merino FJ, Martinez-Ruiz R, Bailo B, Aguilera M, Fuentes I, et al. Molecular genotyping and sub-genotyping of Cryptosporidium spp isolates from symptomatic individuals attending two major public hospitals in Madrid, Spain. Infect Genet Evol. 2016;37:49-56.

37. Alves M, Xiao L, Antunes F, Matos O. Distribution of Cryptosporidium subtypes in humans and domestic and wild ruminants in Portugal. Parasitol Res. 2006;99:287-92.

38. Hu S, Liu Z, Yan F, Zhang Z, Zhang G, Zhang L, et al. Zoonotic and hostadapted genotypes of Cryptosporidium spp., Giardia duodenalis and Enterocytozoon bieneusi in dairy cattle in Hebei and Tianjin, China. Vet Parasitol. 2017;248:68-73.

39. Wang R, Wang H, Sun Y, Zhang L, Jian F, Qi M, et al. Characteristics of Cryptosporidium transmission in preweaned dairy cattle in Henan, China. J Clin Microbiol. 2011;49:1077-82.

40. Zhang W, Wang R, Yang F, Zhang L, Cao J, Zhang X, et al. Distribution and genetic characterizations of Cryptosporidium spp. in pre-weaned dairy calves in northeastern China's Heilongjiang Province. PloS One. 2013;8:e54857.

41. Wang R, Zhao G, Gong Y, Zhang L. Advances and perspectives on the epidemiology of bovine Cryptosporidium in China in the past 30 years. Front Microbiol. 2017;8:1823.

42. Li F, Wang H, Zhang Z, Li J, Wang C, Zhao J, et al. Prevalence and molecular characterization of Cryptosporidium spp and Giardia duodenalis in dairy cattle in Beijing, China. Vet Parasitol. 2016;219:61-5.

43. Jian F, Liu A, Wang R, Zhang S, Qi M, Zhao W, et al. Common occurrence of Cryptosporidium hominis in horses and donkeys. Infect Genet Evol. 2016;43:261-6.

44. Liu H, Shen Y, Yin J, Yuan Z, Jiang Y, Xu Y, et al. Prevalence and genetic characterization of Cryptosporidium, Enterocytozoon, Giardia and Cyclospora in diarrheal outpatients in China. BMC Infect Dis. 2014;14:25.

45. Mukherjee AK, Chowdhury P, Rajendran K, Nozaki T, Ganguly S. Association between Giardia duodenalis and coinfection with other diarrheacausing pathogens in India. BioMed Res Int. 2014;2014:786480.

46. de Lucio A, Amor-Aramendia A, Bailo B, Saugar JM, Anegagrie M, Arroyo $A$, et al. Prevalence and genetic diversity of Giardia duodenalis and Cryptosporidium spp among school children in a rural area of the Amhara region, north-west Ethiopia. PloS ONE. 2016;11:e0159992.

47. Yu F, Amer S, Qi M, Wang R, Wang Y, Zhang S, et al. Multilocus genotyping of Giardia duodenalis isolated from patients in Egypt. Acta Trop. 2019;196:66-71.

48. Naguib D, El-Gohary AH, Roellig D, Mohamed AA, Arafat N, Wang Y, et al. Molecular characterization of Cryptosporidium spp and Giardia duodenalis in children in Egypt. Parasit Vectors. 2018;11:403.

49. Yang J, Song M, Wan Q, Li Y, Lu Y, Jiang Y, et al. Enterocytozoon bieneusi genotypes in children in northeast China and assessment of risk of zoonotic transmission. J Clin Microbiol. 2014;52:4363-7.

50. Zhang W, Ren G, Zhao W, Yang Z, Shen Y, Sun Y, et al. Genotyping of Enterocytozoon bieneusi and subtyping of Blastocystis in cancer patients: 
relationship to diarrhea and assessment of zoonotic transmission. Front Microbiol. 2017:8:1835.

51. Liu H, Jiang Z, Yuan Z, Yin J, Wang Z, Yu B, et al. Infection by and genotype characteristics of Enterocytozoon bieneusi in HIV/AIDS patients from Guangxi Zhuang autonomous region, China. BMC Infect Dis. 2017;17:684.

52. Yu F, Qi M, Zhao Z, Lv C, Wang Y, Wang R, et al. The potential role of synanthropic rodents and flies in the transmission of Enterocytozoon bieneusi on a dairy cattle farm in China. J Eukaryot Microbiol. 2019;66:435-41.

53. Karim MR, Dong H, Li T, Yu F, Li D, Zhang L, et al. Predomination and new genotypes of Enterocytozoon bieneusi in captive nonhuman primates in zoos in China: high genetic diversity and zoonotic significance. PloS ONE. 2015;10:e0117991.
54. Li J, Luo N, Wang C, Qi M, Cao J, Cui Z, et al. Occurrence, molecular characterization and predominant genotypes of Enterocytozoon bieneusi in dairy cattle in Henan and Ningxia, China. Parasit Vectors. 2016;9:142.

55. Qi M, Wang R, Wang H, Jian F, Li J, Zhao J, et al. Enterocytozoon bieneusi genotypes in grazing horses in China and their zoonotic transmission potential. J Eukaryot Microbiol. 2016;63:591-7.

\section{Publisher's Note}

Springer Nature remains neutral with regard to jurisdictional claims in published maps and institutional affiliations.
Ready to submit your research? Choose BMC and benefit from:

- fast, convenient online submission

- thorough peer review by experienced researchers in your field

- rapid publication on acceptance

- support for research data, including large and complex data types

- gold Open Access which fosters wider collaboration and increased citations

- maximum visibility for your research: over 100M website views per year

At BMC, research is always in progress.

Learn more biomedcentral.com/submissions 